\title{
Cofiniteness of local cohomology modules over Noetherian local rings*
}

\author{
Iraj Bagheriyeh ${ }^{\dagger} \quad$ Jafar A'zami Kamal Bahmanpour
}

Dedicated to Professor Thomas Marley

\begin{abstract}
Let $(R, \mathfrak{m})$ be a commutative Noetherian local ring. Let $I$ be an ideal of $R$ and let $M$ be a finitely generated $R$-module of dimension $d \geq 1$. In this paper we consider the $I$-cofiniteness property of the local cohomology module $H_{I}^{d-1}(M)$. More precisely, we prove that the $R$-module $H_{I}^{d-1}(M)$ is I-cofinite if and only if the $R$-module $\operatorname{Hom}_{R}\left(R / I, H_{I}^{d-1}(M)\right)$ is finitely generated. As an immediate consequence of this result, we prove that if $(R, \mathfrak{m})$ is a regular local ring of dimension $d \geq 2$ and $I$ is an ideal of $R$ with $\operatorname{dim} R / I \neq 1$, then $H_{I}^{d-1}(R)=0$ if and only if the $R$-module $\operatorname{Hom}_{R}\left(R / I, H_{I}^{d-1}(R)\right)$ is finitely generated.
\end{abstract}

\section{Introduction}

Throughout this paper, let $R$ denote a commutative Noetherian ring (with identity) and $I$ an ideal of $R$. For an $R$-module $M$, the $i^{\text {th }}$ local cohomology module of $M$ with respect to $I$ is defined as

$$
H_{I}^{i}(M)=\underset{n \geq 1}{\lim } \operatorname{Ext}_{R}^{i}\left(R / I^{n}, M\right)
$$

\footnotetext{
*This research of the authors was been supported by a grant from Islamic Azad UniversityHashtroud branch.

${ }^{\dagger}$ Corresponding author

Received by the editors in July 2013 - In revised form in June 2015.

Communicated by M. Van den Bergh.

2010 Mathematics Subject Classification : 13D45, 14B15, $13 \mathrm{E} 05$.

Key words and phrases : Associated primes, cofinite module, Krull dimension, local cohomology.
} 
Our terminology follows the textbook by Brodmann and Sharp [7] about local cohomology.

It is well-known that if $R$ is a local (Noetherian) ring with maximal ideal $\mathfrak{m}$, then the $R$-module $M$ is Artinian if and only if Supp $M \subseteq\{\mathfrak{m}\}$ and $\operatorname{Ext}_{R}^{j}(R / \mathfrak{m}, M)$ is finitely generated for all $j \geq 0$ (cf. [11, Proposition 1.1]).

Hartshorne [11] introduced the class of cofinite modules, answering in negative a question of Grothendieck (cf. [10, Exposé XIII, Conjecture 1.1]). In fact, Grothendieck conjectured that for any ideal $I$ of $R$ and any finitely generated $R$-module $M$, the module $\operatorname{Hom}_{R}\left(R / I, H_{I}^{i}(M)\right)$ is finitely generated, where $H_{I}^{i}(M)$ is the $i$-th local cohomology module of $M$ with support in $V(I)$, (this is the case when $I=\mathfrak{m}$, the maximal ideal in a local ring, since the modules $H_{\mathfrak{m}}^{i}(M)$ are Artinian). But soon Hartshorne was able to present a counterexample (see [11] for details and proof) which shows that this conjecture is false even when $R$ is regular, and he defined an $R$-module $M$ to be $I$-cofinite if the support of $M$ is contained in $V(I)$ and $\operatorname{Ext}_{R}^{j}(R / I, M)$ is finitely generated for all $j$ and asked the following questions:

(i) For which rings $R$ and ideals $I$ are the modules $H_{I}^{i}(M)$ I-cofinite for all $i$ and all finitely generated modules $M$ ?

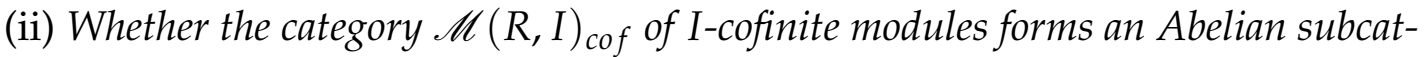
egory of the category of all $R$-modules? That is, if $f: M \longrightarrow N$ is an R-homomorphism of I-cofinite modules, are ker $f$ and coker $f$ I-cofinite?

With respect to the question (i), Hartshorne in [11] and later Chiriacescu in [8] showed that if $R$ is a complete regular local ring and $I$ is a prime ideal with $\operatorname{dim} R / I=1$, then $H_{I}^{i}(M)$ is $I$-cofinite for any finitely generated $R$-module $M$.

Also, Delfino and Marley [9] and Yoshida [22] have eliminated the complete hypothesis entirely. Finally, the local condition on the ring has been removed in [3].

With respect to the question (ii), Hartshorne gave an example to show that the question (ii) has not an affirmative answer in general. However, he proved that if $I$ is a prime ideal of dimension one in a complete regular local ring $R$, then the answer to his question is yes. In [9], Delfino and Marley extended this result to arbitrary complete local rings. Recently, Kawasaki [13], using an spectral sequence argument, generalized the Delfino and Marley's result for an arbitrary ideal $I$ of dimension one in a local ring $R$. Finally, Melkersson in [18] proved that Hartshorne's question is true for all ideals of dimension one of any arbitrary Noetherian ring $R$.

More recently, in [5] it is shown that Hartshorne's question is true for $\mathscr{C}^{1}(R, I)_{\text {cof }}$, the category of all $I$-cofinite $R$-modules $M$ with $\operatorname{dim} M \leq 1$, for all ideals $I$ in a commutative Noetherian ring $R$. The proof of this result is based 
on [5, Proposition 2.6], which states that in order to deduce the I-cofiniteness for a module $M$ with $\operatorname{dim} M \leq 1$ and $\operatorname{Supp} M \subseteq V(I)$, it suffices that the $R$-modules $\operatorname{Hom}_{R}(R / I, M)$ and $\operatorname{Ext}_{R}^{1}(R / I, M)$ be finitely generated. In particular, if $\operatorname{dim} R / I=1$ and $\operatorname{Supp} M \subseteq V(I)$ then $M$ is I-cofinite if (and only if) $\operatorname{Hom}_{R}(R / I, M)$ and $\operatorname{Ext}_{R}^{1}(R / I, M)$ are finitely generated. Also, in [4], Bahmanpour et al as a generalization of [18, Theorem 2.3] have shown that if $I$ is an ideal of a Noetherian local ring $(R, \mathfrak{m})$ with $\operatorname{dim} R / I=2$ and $M$ is an $R$-module such that Supp $M \subseteq V(I)$ then $M$ is $I$-cofinite if (and only if) the $R$-modules $\operatorname{Hom}_{R}(R / I, M), \operatorname{Ext}_{R}^{1}(R / I, M)$, and $\operatorname{Ext}_{R}^{2}(R / I, M)$ are finitely generated.

Pursuing this point of view further we establish some results concerning the cofiniteness of local cohomology modules. In fact, as the main result of this paper, we present a criterion for $I$-cofiniteness of the local cohomology module $H_{I}^{d-1}(M)$, where $I$ is an arbitrary ideal of a Noetherian local ring $(R, \mathfrak{m})$ and $M$ is a finitely generated $R$-module of dimension $d \geq 1$. More precisely, it is shown that the $R$-module $H_{I}^{d-1}(M)$ is $I$-cofinite if and only if the $R$-module $\operatorname{Hom}_{R}\left(R / I, H_{I}^{d-1}(M)\right)$ is finitely generated.

For each $R$-module $L$, we denote by $\operatorname{Assh}_{R} L$ the set $\left\{\mathfrak{p} \in \operatorname{Ass}_{R} L: \operatorname{dim} R / \mathfrak{p}=\right.$ $\operatorname{dim} L\}$. Also, for any ideal $\mathfrak{a}$ of $R$, we denote $\{\mathfrak{p} \in \operatorname{Spec} R: \mathfrak{p} \supseteq \mathfrak{a}\}$ by $V(\mathfrak{a})$. Finally, for any ideal $\mathfrak{b}$ of $R$, the radical of $\mathfrak{b}$, denoted by $\operatorname{Rad}(\mathfrak{b})$, is defined to be the set $\left\{x \in R: x^{n} \in \mathfrak{b}\right.$ for some $\left.n \in \mathbb{N}\right\}$. We refer the reader to [7] for more details about local cohomology. Also, for any unexplained notation and terminology we refer the reader to [7] and [17].

\section{Preliminaries}

The purpose of this section is to provide a technical result which will be useful in the next section.

Lemma 2.1. Let $R$ be a Noetherian ring. Let $I$ be an ideal of $R$ such that $\operatorname{dim} R / I=1$ and let $M$ be a non-zero $R$-module of dimension $n \leq 1$, such that the $R$-modules $\operatorname{Hom}_{R}(R / I, M)$ and $\operatorname{Ext}_{R}^{1}(R / I, M)$ are finitely generated. Then for each $i \geq 0$, the $R$-module $\operatorname{Ext}_{R}^{i}(R / I, M)$ is finitely generated.

Proof. Follows from [4, Theorem 2.5].

Now we are prepared to state and prove the main result of this section, which plays a key role in the proof of the main result of this paper.

Theorem 2.2. Let $(R, \mathfrak{m})$ be a Noetherian local ring and let $I$ be an ideal of $R$. Let $M$ be a finitely generated $R$-module of dimension $d \geq 1$ with $H_{I}^{d-1}(M) \neq 0$. Let $J:=\cap_{\mathfrak{p} \in \operatorname{Supp} H_{I}^{d-1}(M)} \mathfrak{p}$. Then the following statements are equivalent:

(i) The R-module $H_{I}^{d-1}(M)$ is J-cofinite.

(ii) The R-module $\operatorname{Hom}_{R}\left(R / J, H_{I}^{d-1}(M)\right)$ is finitely generated. 
Proof. (i) $\Rightarrow$ (ii) Is clear.

(ii) $\Rightarrow$ (i) In view of [15, Corollary 2.5], the set Supp $H_{I}^{d-1}(M)$ is finite. So we have $\operatorname{dim} R / J \leq 1$. In the case where $\operatorname{dim} R / J=0$ there is nothing to prove. Because, in this situation $H_{I}^{d-1}(M)$ is Artinian and $J=\mathfrak{m}$. Now, suppose that $\operatorname{dim} R / J=1$. If we have $\operatorname{dim} R / I=1$ then in view of [9, Theorem 1], the $R$-module $H_{I}^{d-1}(M)$ is $I$-cofinite and so as $I \subseteq J$ it follows from [9, Corollary $1]$, that the $R$-module $H_{I}^{d-1}(M)$ is $J$-cofinite. So, we may assume $\operatorname{dim} R / I \geq 2$. Then as $\operatorname{dim} R / \operatorname{Rad}(I) \geq 2$ it follows that there is an element $x_{1} \in(J \backslash \operatorname{Rad}(I))$. By [21, Corollary 3.5], there is an exact sequence

$$
0 \longrightarrow H_{R x_{1}}^{1}\left(H_{I}^{d-2}(M)\right) \longrightarrow H_{I+R x_{1}}^{d-1}(M) \stackrel{f_{1}}{\longrightarrow} H_{I}^{d-1}(M) \rightarrow 0,
$$

(Note that the $R$-module $H_{I}^{d-1}(M)$ is $J$-torsion and hence from the hypothesis $x_{1} \in J$, we have $\left.H_{R x_{1}}^{0}\left(H_{I}^{d-1}(M)\right) \simeq H_{I}^{d-1}(M)\right)$. Now, if $\operatorname{dim} R /\left(I+R x_{1}\right)=1$ then in view of [9, Theorem 1], the $R$-module $H_{I+R x_{1}}^{d-1}(M)$ is $I+R x_{1}$-cofinite. Since, $\operatorname{Rad}\left(I+R x_{1}\right) \subseteq \operatorname{Rad}(J)=J$ it follows from [9, Corollary 1] that the $R$-module $\operatorname{Ext}_{R}^{j}\left(R / J, H_{I+R x_{1}}^{d-1}(M)\right)$ is finitely generated for all $j$. So, it follows from the exact sequence (2.2.1) that the $R$-modules

$$
\operatorname{Hom}_{R}\left(R / J, H_{R x_{1}}^{1}\left(H_{I}^{d-2}(M)\right)\right) \text { and } \operatorname{Ext}_{R}^{1}\left(R / J, H_{R x_{1}}^{1}\left(H_{I}^{d-2}(M)\right)\right)
$$

are finitely generated. Since, $\operatorname{dim} H_{R x_{1}}^{1}\left(H_{I}^{d-2}(M)\right) \leq 1$ it follows from Lemma 2.1, that the $R$-modules $\operatorname{Ext}_{R}^{j}\left(R / J, H_{R x_{1}}^{1}\left(H_{I}^{d-2}(M)\right)\right)$ are finitely generated for all $j$. Now, from the exact sequence (2.2.1) it follows that the $R$-module $H_{I}^{d-1}(M)$ is $J$-cofinite. So, we may assume $\operatorname{dim} R /\left(I+R x_{1}\right) \geq 2$. Let $J_{1}=\cap_{\mathfrak{p} \in \operatorname{Supp} H_{I+R x_{1}}^{d-1}(M)} \mathfrak{p}$. Then $J_{1} \subseteq J$.

Since, $\operatorname{dim} R / J_{1}=1$ it follows that there is an element $x_{2} \in\left(J_{1} \backslash \operatorname{Rad}\left(I+R x_{1}\right)\right)$. Let $I_{1}:=I+R x_{1}$. By [21, Corollary 3.5] there is an exact sequence

$$
0 \longrightarrow H_{R x_{2}}^{1}\left(H_{I_{1}}^{d-2}(M)\right) \longrightarrow H_{I_{1}+R x_{2}}^{d-1}(M) \stackrel{f_{2}}{\longrightarrow} H_{I_{1}}^{d-1}(M) \rightarrow 0
$$

(Note that the $R$-module $H_{I_{1}}^{d-1}(M)$ is $J_{1}$-torsion and hence from the hypothesis $x_{2} \in J_{1}$, we have $\left.H_{R x_{2}}^{0}\left(H_{I_{1}}^{d-1}(M)\right) \simeq H_{I_{1}}^{d-1}(M)\right)$. Now, if $\operatorname{dim} R /\left(I_{1}+R x_{2}\right)=1$, then in view of [9, Theorem 1], the $R$-module $H_{I_{1}+R x_{2}}^{d-1}(M)$ is $\left(I_{1}+R x_{2}\right)$-cofinite. So, as $\operatorname{Rad}\left(I+R x_{2}\right) \subseteq \operatorname{Rad}\left(J_{1}\right)=J_{1} \subseteq J$ it follows from [9, Corollary 1] that the $R$-module

$$
\operatorname{Ext}_{R}^{j}\left(R / J, H_{I_{1}+R x_{2}}^{d-1}(M)\right)
$$

is finitely generated for all $j$. Therefore, from the exact

$$
0 \rightarrow K_{1} \rightarrow H_{I_{1}+R x_{2}}^{d-1}(M) \stackrel{f_{1} \circ f_{2}}{\longrightarrow} H_{I}^{d-1}(M) \rightarrow 0, \quad(2.2 .2), \quad\left(\text { where, } K_{1}=\operatorname{ker} f_{1} \circ f_{2}\right)
$$

it follows that the $R$-modules $\operatorname{Hom}_{R}\left(R / J, K_{1}\right)$ and $\operatorname{Ext}_{R}^{1}\left(R / J, K_{1}\right)$ are finitely generated. Since, $\operatorname{dim} K_{1} \leq 1$ it follows from Lemma 2.1 that the $R$-module 
$\operatorname{Ext}_{R}^{j}\left(R / J, K_{1}\right)$ is finitely generated for all $j$. Now, it follows from the exact sequence (2.2.2), that $H_{I}^{d-1}(M)$ is $J$-cofinite.

So, we may assume $\operatorname{dim} R /\left(I_{1}+R x_{2}\right) \geq 2$. Let $J_{2}=\cap_{\mathfrak{p} \in \operatorname{Supp} H_{I_{1}+R x_{2}}^{d-1}(M)} \mathfrak{p}$.

Then $J_{2} \subseteq J_{1} \subseteq J$. Since, $\operatorname{dim} R / J_{2}=1$ it follows that there is an element $x_{3} \in\left(J_{2} \backslash \operatorname{Rad}\left(I_{1}+R x_{2}\right)\right)$. Let $I_{2}:=I_{1}+R x_{2}$. By [21, Corollary 3.5], there is an exact sequence

$$
0 \longrightarrow H_{R x_{3}}^{1}\left(H_{I_{2}}^{d-2}(M)\right) \longrightarrow H_{I_{2}+R x_{3}}^{d-1}(M) \stackrel{f_{3}}{\longrightarrow} H_{I_{2}}^{d-1}(M) \rightarrow 0 .
$$

(Note that the $R$-module $H_{I_{2}}^{d-1}(M)$ is $J_{2}$-torsion and hence from the hypothesis $x_{3} \in J_{2}$, we have $H_{R x_{3}}^{0}\left(H_{I_{2}}^{d-1}(M)\right) \simeq H_{I_{2}}^{d-1}(M)$ ). Now, applying the method used above, from the exact sequence

$$
0 \rightarrow K_{2} \rightarrow H_{I_{2}+R x_{3}}^{d-1}(M) \stackrel{f_{1} \circ f_{2} \circ f_{3}}{\longrightarrow} H_{I}^{d-1}(M) \rightarrow 0, \quad\left(\text { where, } K_{2}=\operatorname{ker} f_{1} \circ f_{2} \circ f_{3}\right)
$$

it follows that if $\operatorname{dim} R /\left(I_{2}+R x_{3}\right)=1$, then the $R$-module $H_{I}^{d-1}(M)$ is J-cofinite. Proceeding in the same way, as $R$ is Noetherian it follows that after finitely many steps repeating this argument we can find an ideal $I_{k}$ and an element $x_{k+1} \in J_{k}$, with $I \subset I_{1} \subset \cdots \subset I_{k}$ such that $\operatorname{dim} R /\left(I_{k}+R x_{k+1}\right)=1$, which implies the $R$-module $H_{I}^{d-1}(M)$ is $J$-cofinite.

The following result is a consequence of Theorem 2.2.

Corollary 2.3. Let $(R, \mathfrak{m})$ be a Noetherian local ring. Let $I$ be an ideal of $R$ and let $M$ be $a$ finitely generated $R$-module of dimensiond $\geq 1$. If the $R$-module $\operatorname{Hom}_{R}\left(R / I, H_{I}^{d-1}(M)\right)$ is finitely generated, then the R-module $H_{I}^{d-1}(M)$ is J-cofinite, where :

$$
J:=\bigcap_{\mathfrak{p} \in \operatorname{Supp} H_{I}^{d-1}(M)} \mathfrak{p} .
$$

Proof. As $I \subseteq J$ it follows that the $R$-module $\operatorname{Hom}_{R}\left(R / J, H_{I}^{d-1}(M)\right)$ is finitely generated and so the assertion follows from Theorem 2.2.

\section{The Main Result}

Let $(R, \mathfrak{m})$ be a Noetherian local ring. Let $I$ an ideal of $R$ and let $M$ be a finitely generated $R$-module of dimension $d \geq 1$. In [15, Corollary 2.5], it has shown that the set Supp $H_{I}^{d-1}(M)$ is finite and so $\operatorname{dim} H_{I}^{d-1}(M) \leq 1$. So, by [18, Theorem 2.3] or [5, Proposition 2.6] we know that the $R$-module $H_{I}^{d-1}(M)$ is $I$-cofinite, if and only if the $R$-modules $\operatorname{Hom}_{R}\left(R / I, H_{I}^{d-1}(M)\right)$ and $\operatorname{Ext}_{R}^{1}\left(R / I, H_{I}^{d-1}(M)\right)$ are finitely generated. Our main purpose in this section is to show that in this statement, the condition on the $R$-module $\operatorname{Ext}_{R}^{1}\left(R / I, H_{I}^{d-1}(M)\right)$ can be omitted.

Lemma 3.1. Let $\mathfrak{a} \subseteq \mathfrak{b}$ be ideals of a Noetherian ring $R$, and let $M$ be an $R$-module such that the $R$-modules $\operatorname{Ext}_{R}^{j}\left(R / \mathfrak{b}, H_{\mathfrak{a}}^{i}(M)\right)$ are finitely generated for all $i$ and $j$ (respectively for $i \leq n$ and all $j)$. Then the $R$-module $\operatorname{Ext}_{R}^{i}(R / \mathfrak{b}, M)$ is finitely generated for all $i$ (respectively for all $i \leq n$ ). 
Proof. See [1, Lemma 2.1].

Now, we are ready to state and prove the main result of this paper.

Lemma 3.2. Let $(R, \mathfrak{m})$ be a Noetherian local ring. Let $I$ be an ideal of $R$ and let $M$ be a finitely generated $R$-module of dimension $d \geq 1$. Then the following statements are equivalent:

(i) The R-module $H_{I}^{d-1}(M)$ is I-cofinite.

(ii) The $R$-module $\operatorname{Hom}_{R}\left(R / I, H_{I}^{d-1}(M)\right)$ is finitely generated.

Proof. (i) $\Rightarrow$ (ii) Is clear.

(ii) $\Rightarrow$ (i) First we consider the assertion in the following situations:

1 - $\operatorname{dim} R / I \leq 1$. In this situation the assertion follows from [9, Theorem 1].

2- $\operatorname{dim} H_{I}^{d-1}(M)=0$. In this situation the assertion follows from [19, Proposition 4.1].

Now, we may assume $\operatorname{dim} R / I \geq 2$ and $\operatorname{dim} H_{I}^{d-1}(M)>0$. Also, due to faithful flatness of $\widehat{R}$ (the m-adic completion of $R$ ), we may assume $R$ is complete. Then by Corollary 2.3 there exists an ideal $J:=\bigcap_{\mathfrak{p} \in \operatorname{Supp} H_{I}^{d-1}(M)} \mathfrak{p}$ such that $\operatorname{dim} R / J=1$ and $H_{I}^{d-1}(M)$ is $J$-cofinite and $I \subseteq J$. Now using induction on $k=\operatorname{dim} N \leq$ $\operatorname{dim} R / I$ we show that for any finitely generated module $N$ with Supp $N \subseteq V(I)$ the $R$-modules $\operatorname{Ext}_{R}^{j}\left(N, H_{I}^{d-1}(M)\right)$ are finitely generated for all $j$. To do this, first let $k=1$. First we show that for each $\mathfrak{p} \in V(I)$ with $\operatorname{dim} R / \mathfrak{p}=1$ the $R$-modules $\operatorname{Ext}_{R}^{j}\left(R / \mathfrak{p}, H_{I}^{d-1}(M)\right)$ are finitely generated. But if $\mathfrak{p} \in \operatorname{Supp} H_{I}^{d-1}(M)$ then as $J \subseteq \mathfrak{p}$ the assertion follows from Theorem 2.2 and [9, Corollary 1]. Now suppose that $\mathfrak{p} \notin \operatorname{Supp} H_{I}^{d-1}(M)$. Then there exists

$$
x \in\left[\mathfrak{p} \backslash\left(\cup_{\mathfrak{q} \in \operatorname{mAss}_{R} H_{I}^{d-1}(M)} \mathfrak{q}\right)\right] .
$$

Then we have $\Gamma_{R x}\left(H_{I}^{d-1}(M)\right)=\Gamma_{\mathfrak{m}}\left(H_{I}^{d-1}(M)\right)$. Since the $R$-module

$$
\operatorname{Hom}_{R}\left(R / \mathfrak{p}, \Gamma_{\mathfrak{m}}\left(H_{I}^{d-1}(M)\right)\right)
$$

is finitely generated it follows from [19, Proposition 4.1] that $\Gamma_{R x}\left(H_{I}^{d-1}(M)\right)$ is $\mathfrak{p}$-cofinite. By [21, Corollary 3.5] there is an exact sequence

$$
0 \longrightarrow H_{R x}^{1}\left(H_{I}^{d-1}(M)\right) \longrightarrow H_{I+R x}^{d}(M) \longrightarrow H_{R x}^{0}\left(H_{I}^{d}(M)\right) \rightarrow 0 .
$$

By [20, Proposition 5.1], the $R$-module $H_{I+R x}^{d}(M)$ is Artinian $(I+R x)$-cofinite. So from [9, Corollary 1] and [19, Proposition 4.1], we can deduce that $H_{R x}^{1}\left(H_{I}^{d-1}(M)\right)$ is Artinian p-cofinite. Moreover, by [7, Exercise 2.1.9] we have

$$
H_{R x}^{1}\left(H_{I}^{d-1}(M)\right) \cong H_{I+R x}^{1}\left(H_{I}^{d-1}(M)\right) \text { and } H_{R x}^{0}\left(H_{I}^{d-1}(M)\right) \cong H_{I+R x}^{0}\left(H_{I}^{d-1}(M)\right),
$$

and by [7, Corollary 3.3.3], we have $H_{I+R x}^{k}\left(H_{I}^{d-1}(M)\right) \cong H_{R x}^{k}\left(H_{I}^{d-1}(M)\right)=$ 0 , for all $k \geq 2$. Hence, from the Lemma 3.1 it follows that the $R$-module $\operatorname{Ext}_{R}^{j}\left(R / \mathfrak{p}, H_{I}^{\bar{d}-1}(M)\right)$ is finitely generated for all $j \geq 0$. On the other hand, 
as the $R$-module $\operatorname{Hom}_{R}\left(R / \mathfrak{m}, H_{I}^{d-1}(M)\right)$ is finitely generated, it follows from [6, Theorem 2.12], that the $R$-modules

$$
\operatorname{Ext}_{R}^{j}\left(R / \mathfrak{m}, H_{I}^{d-1}(M)\right)
$$

are finitely generated for all $j$. Now, from [17, Theorem 6.4], it follows that for any finitely generated $R$-module $N$ of dimension $n \leq 1$ and $\operatorname{Supp} N \subseteq V(I)$ the $R$-modules $\operatorname{Ext}_{R}^{j}\left(N, H_{I}^{d-1}(M)\right)$ are finitely generated for all $j$. Now let $1<k \leq$ $\operatorname{dim} R / I$ the the case $k-1$ is settled. First let $\mathfrak{p} \in V(I)$ and $\operatorname{dim} R / \mathfrak{p}=k$. Let $x \in(\mathfrak{m} \backslash \mathfrak{p})$. By inductive hypothesis the $R$-modules $\operatorname{Ext}_{R}^{j}\left(R /(\mathfrak{p}+R x), H_{I}^{d-1}(M)\right)$ are finitely generated for all $j \geq 0$.

Now from the exact sequence

$$
0 \rightarrow R / \mathfrak{p} \stackrel{x}{\rightarrow} R / \mathfrak{p} \rightarrow R /(\mathfrak{p}+R x) \rightarrow 0,
$$

we get the following exact sequence

$$
\begin{aligned}
\operatorname{Ext}_{R}^{i}\left(R /(\mathfrak{p}+R x), H_{I}^{d-1}(M)\right) \rightarrow \operatorname{Ext}_{R}^{i}(R / \mathfrak{p}, & \left.H_{I}^{d-1}(M)\right) \\
& \stackrel{x}{\rightarrow} \operatorname{Ext}_{R}^{i}\left(R / \mathfrak{p}, H_{I}^{d-1}(M)\right),
\end{aligned}
$$

Let $L:=\operatorname{Ext}_{R}^{i}\left(R / \mathfrak{p}, H_{I}^{d-1}(M)\right)$. From the exact sequence (3.2.1) we conclude that the $R$-module $0::_{L} x$ is finitely generated. But as the $R$-module $\operatorname{Hom}_{R}(R / \mathfrak{m}$, $\left.H_{I}^{d-1}(M)\right)$ is finitely generated, it follows from [4, Theorem 2.12], that the $R$-module $L$ is minimax. Because in this situation the $R$-module $H_{I}^{d-1}(M)$ is $I$-cominimax. So there is a finitely generated submodule $K$ of $L$ such that $L / K$ is Artinian. We claim that $L$ is finitely generated. Suppose the contrary be true. Then $L / K$ is not finitely generated. From the fact that the $R$-module $0:_{L} x$ is finitely generated, it follows that the $R$-module $0:_{L / K} x$ is finitely generated. So $L / K$ is $R x$-cofinite. Since, by hypothesis $R$ is complete from [20, Theorem 1.6], it follows that $\operatorname{dim} R /(R x+\operatorname{Ann} L / K)=0$ and $\operatorname{sodim} R / \operatorname{Ann} L / K=1$. Moreover as $K$ is finitely generated and Supp $K \subseteq \operatorname{Supp} L \subseteq \operatorname{Supp} H_{I}^{d-1}(M)$ it follows that $\operatorname{dim} R / \operatorname{Ann} K=\operatorname{dim} K=1$. Therefore, since $(\operatorname{Ann} K)(\operatorname{Ann} L / K) \subseteq \operatorname{Ann} L$ it follows that $\operatorname{dim} R / \operatorname{Ann} L=1$. Next there is an element $z \in[\operatorname{Ann} L \backslash \mathfrak{p}]$. Now from the exact sequence

$$
0 \rightarrow R / \mathfrak{p} \stackrel{z}{\rightarrow} R / \mathfrak{p} \rightarrow R /(\mathfrak{p}+R z) \rightarrow 0,
$$

we get the following exact sequence

$$
\operatorname{Ext}_{R}^{i}\left(R /(\mathfrak{p}+R z), H_{I}^{d-1}(M)\right) \rightarrow \operatorname{Ext}_{R}^{i}\left(R / \mathfrak{p}, H_{I}^{d-1}(M)\right) \stackrel{z}{\rightarrow} \operatorname{Ext}_{R}^{i}\left(R / \mathfrak{p}, H_{I}^{d-1}(M)\right)
$$

But this exact sequence induces the following exact sequence

$$
\operatorname{Ext}_{R}^{i}\left(R /(\mathfrak{p}+R z), H_{I}^{d-1}(M)\right) \rightarrow \operatorname{Ext}_{R}^{i}\left(R / \mathfrak{p}, H_{I}^{d-1}(M)\right) \rightarrow 0 .
$$

Since by inductive hypothesis the module $\operatorname{Ext}_{R}^{i}\left(R /(\mathfrak{p}+R z), H_{I}^{d-1}(M)\right)$ is finitely generated it follows that the $R$-module $\operatorname{Ext}_{R}^{i}\left(R / \mathfrak{p}, H_{I}^{d-1}(M)\right)$ is finitely generated, which is a contradiction. Since $i$ is arbitrary it follows that $\operatorname{Ext}_{R}^{j}(R / \mathfrak{p}$, 
$\left.H_{I}^{d-1}(M)\right)$ is finitely generated for all $j \geq 0$. Now from [17, Theorem 6.4], it follows that for any finitely generated module $N$ of dimension $n \leq k$ and Supp $N \subseteq V(I)$ the $R$-modules $\operatorname{Ext}_{R}^{j}\left(N, H_{I}^{d-1}(M)\right)$ are finitely generated for all $j$. This completes the inductive step.

Now as an application of Theorem 3.2, we prove the following result, which is an immediate consequence of [12, Theorem 2.3].

Theorem 3.3. Let $(R, \mathfrak{m})$ be a Noetherian regular local ring of dimension $d \geq 2$ and let $I$ be an ideal of $R$ with $\operatorname{dim} R / I \geq 2$. Then the following statements are equivalent:

(i) $H_{I}^{d-1}(R)=0$.

(ii) The R-module $\operatorname{Hom}_{R}\left(R / I, H_{I}^{d-1}(R)\right)$ is finitely generated.

Proof. (i) $\Rightarrow$ (ii) Is clear.

(ii) $\Rightarrow$ (i) Due to faithful flatness of $\widehat{R}$ (the $\mathfrak{m}$-adic completion of $R$ ), we may assume $R$ is complete. Now, suppose that the $R$-module $\operatorname{Hom}_{R}\left(R / I, H_{I}^{d-1}(R)\right)$ is finitely generated, but $H_{I}^{d-1}(R) \neq 0$. Then we have $0 \leq \operatorname{dim} H_{I}^{d-1}(R) \leq 1$. First suppose that $\operatorname{dim} H_{I}^{d-1}(R)=0$. Then by [19, Proposition 4.1], the $R$-module $H_{I}^{d-1}(R)$ is Artinian $I$-cofinite. Since by hypothesis $R$ is complete, form [20, Theorem 1.6], it follows that $\operatorname{Rad}\left(I+\operatorname{Ann} H_{I}^{d-1}(R)\right)=\mathfrak{m}$. Now as $\operatorname{dim} R / I \geq 2$ it follows that Ann $H_{I}^{d-1}(R) \neq 0$. Also by the Lichtenbaum-Hartshorne Vanishing Theorem we have $H_{I}^{d}(R)=0$. Let $0 \neq x \in \operatorname{Ann} H_{I}^{d-1}(R)$. Then from the exact sequence

$$
0 \rightarrow R \stackrel{x}{\rightarrow} R \rightarrow R / R x \rightarrow 0
$$

we get the following isomorphism $0 \neq H_{I}^{d-1}(R) \cong H_{I}^{d-1}(R / x R)$. So by the Lichtenbaum-Hartshorne Vanishing Theorem, there exists $\mathfrak{p} \in \operatorname{Assh}_{R} R / x R$ such that $\operatorname{Rad}(I+\mathfrak{p})=\mathfrak{m}$. But $R$ is a U.F.D and so $\mathfrak{p}=R z$ is a principal ideal. This implies that $\operatorname{dim} R / I \leq 1$, which is a contradiction.

Now suppose that $\operatorname{dim} H_{I}^{d-1}(R)=1$. Since by Theorem 3.2, the $R$-module $H_{I}^{d-1}(R)$ is $I$-cofinite, it follows from [14, Theorem 2.9], that $H_{\mathfrak{m}}^{1}\left(H_{I}^{d-1}(R)\right) \neq 0$. In view of [15, Corollary 2.5], the set Supp $H_{I}^{d-1}(R)$ is finite. So there is an element $x \in \mathfrak{m}$ such that $x \notin \cup_{\mathfrak{q} \in \mathrm{mAss}_{R} H_{I}^{d-1}(R)} \mathfrak{q}$. Then there is an exact sequence

$$
0 \longrightarrow H_{R x}^{1}\left(H_{I}^{d-1}(R)\right) \longrightarrow H_{I+R x}^{d}(R) \longrightarrow H_{R x_{1}}^{0}\left(H_{I}^{d}(R)\right) \rightarrow 0 .
$$

Let $J:=\cap_{\mathfrak{p} \in \operatorname{Supp} H_{I}^{d-1}(M)} \mathfrak{p}$. Then $H_{I}^{d-1}(R)$ is $J$-torsion and $\operatorname{Rad}(J+R x)=\mathfrak{m}$. So it is easy to see that $H_{R x}^{1}\left(H_{I}^{d-1}(R)\right)=H_{\mathfrak{m}}^{1}\left(H_{I}^{d-1}(R)\right) \neq 0$, which implies that $H_{I+R x}^{d}(R) \neq 0$. So by Lichtenbaum-Hartshorne Vanishing Theorem we have $\operatorname{Rad}(I+R x)=\mathfrak{m}$. Therefore $\operatorname{dim} R / I=1$, which is a contradiction.

Remark 3.4. In [2, Theorem 3.8], it has been shown that if $(R, \mathfrak{m})$ is a Noetherian local ring of dimension $d \geq 3$, and $x_{1}, \ldots, x_{d}$ is a system of parameters for $R$ and $I=\left(x_{1}\right) \cap\left(x_{2}, \ldots, x_{d}\right)$ then the $R$-module $\operatorname{Hom}_{R}\left(R / I, H_{I}^{d-1}(R)\right)$ is not finitely 
generated. In particular, $H_{I}^{d-1}(R)$ does not satisfy in the equivalent conditions of Theorem 3.2. Also in [11], it has been shown that if

$$
I:=(x, y) R \subseteq R:=k[x, y, z, w] /(x z-y w),
$$

then $\mu^{0}\left(\mathfrak{m}, H_{I}^{2}(R)\right)=\infty$ for $\mathfrak{m}:=(x, y, z, w) R$. In particular, $\left(R_{\mathfrak{m}}, \mathfrak{m} R_{\mathfrak{m}}\right)$ is a Noetherian local ring of dimension 3 such that the $R$-module

$$
\operatorname{Hom}_{R_{\mathfrak{m}}}\left(R_{\mathfrak{m}} / \mathfrak{m} R_{\mathfrak{m}}, H_{I R_{\mathfrak{m}}}^{2}\left(R_{\mathfrak{m}}\right)\right)
$$

is not finitely generated. In particular, $H_{I R_{\mathfrak{m}}}^{2}\left(R_{\mathfrak{m}}\right)$ does not satisfy in the equivalent conditions of Theorem 2.2.

\section{Acknowledgments}

The authors are deeply grateful to the referee for his/her careful reading of the paper and valuable suggestions. Also, the authors would like to thank from the Hashtroud branch, Islamic Azad University for its financial support.

\section{References}

[1] N. Abazari and K. Bahmanpour, On the finiteness of Bass numbers of local cohomology modules, J. Alg. Appl. 10 (2011), 783-791.

[2] K. Bahmanpour and R. Naghipour, Associated primes of local cohomology modules and Matlis duality, J. Algebra 320 (2008), 2632-2641.

[3] K. Bahmanpour and R. Naghipour, Cofiniteness of local cohomology modules for ideals of small dimension, J. Algebra 321 (2009), 1997-2011.

[4] K. Bahmanpour, R. Naghipour and M. Sedghi, Cofiniteness with respect to ideals of small dimensions, Algebr. Represent. Theory 18(2015), 369-379.

[5] K. Bahmanpour, R. Naghipour and M. Sedghi, On the category of cofinite modules which is Abelian, Proc. Amer. Math. Soc. 142 (2014), 1101-1107.

[6] K. Bahmanpour, R. Naghipour and M. Sedghi, On the finiteness of Bass numbers of local cohomology modules and cominimaxness, Houston J. Math. 40 (2014), 319-337.

[7] M.P. Brodmann and R.Y. Sharp, Local cohomology; an algebraic introduction with geometric applications, Cambridge University Press, Cambridge, UK, 1998.

[8] G. Chiriacescu, Cofiniteness of local cohomology modules, Bull. London Math. Soc. 32 (2000), 1-7.

[9] D. Delfino and T. Marley, Cofinite modules and local cohomology, J. Pure Appl. Algebra 121 (1997), 45-52. 
[10] A. Grothendieck, Cohomologie local des faisceaux coherents et théorémes de lefschetz locaux et globaux (SGA2), North-Holland, Amsterdam,1968.

[11] R. Hartshorne, Affine duality and cofiniteness, Invent. Math. 9 (1970), 145-164.

[12] C. Huneke, J. Koh, Cofiniteness and vanishing of local cohomology modules, Math. Proc. Cambridge Philos. Soc. 110 (1991), 421-429.

[13] K.-I. Kawasaki, On a category of cofinite modules which is Abelian, Math. Z. 269 (2011), 587-608.

[14] A. Mafi, Some results on local cohomology modules, Arch. Math. 87 (2006), 211216.

[15] T. Marley, The associated primes of local cohomology modules over rings of small dimension, Manuscripta Math. 104 (2001), 519-525.

[16] T. Marley and J.C. Vassilev, Cofiniteness and associated primes of local cohomology modules, J. Algebra 256 (2002), 180-193.

[17] H. Matsumura, Commutative ring theory, Cambridge University Press, Cambridge, UK, 1986.

[18] L. Melkersson, Cofiniteness with respect to ideals of dimension one, J. Algebra 372 (2012), 459-462.

[19] L. Melkersson, Modules cofinite with respect to an ideal, J. Algebra, 285 (2005), 649-668.

[20] L. Melkersson, Properties of cofinite modules and application to local cohomology, Math. Proc. Cambridge Philos. Soc., 125 (1999), 417-423.

[21] P. Schenzel, Proregular sequences, local cohomology, and completion, Math. Scand. 92 (2003), 161-180.

[22] K.I. Yoshida, Cofiniteness of local cohomology modules for ideals of dimension one, Nagoya Math. J. 147 (1997), 179-191.

Department of Mathematics, Hashtroud branch,

Islamic Azad University,

Hashtroud, Iran.

email:Ir_ba2004@yahoo.com

Department of Mathematics, Faculty of Mathematical Sciences,

University of Mohaghegh Ardabili,

56199-11367, Ardabil, Iran.

emails:jafar.azami@gmail.com, bahmanpour.k@gmail.com 